\title{
ZUR WANDLUNG DES SYSTEMS DER KURZEN VOKALE DES UNGARISCHEN IN DER URUNGARISCHEN UND DER ALTUNGARISCHEN ZEIT
}

\author{
ATTILA HEGEDÜS
}

\begin{abstract}
Auszug
Als Ausgangspunkt der Wandlung der kurzen Vokale des Ungarischen in der altungarischen Zeit bezeichnet István Kenesei die Entwicklung des $i$ in zwei Richtungen $(i \underset{i}{i} u, \underline{i}>i)$, wo die Entwicklung das ganze Kurzvokalsystem veränderte. Nach der Kritik des Keneseis Modells versucht der Autor auf der Traditionen der Literatur zur ungarischen Lautgeschichte ein anderes Modell aufzustellen. Die Grundlage für diese Betrachtungsweise liefern die Betonungsverhältnisse des Ungarischen, und ihre Grundthese besagt, dass $a$ und $\ddot{o}$ zu Beginn der altungarischen Zeit bereits existierten. In der urungarischen Zeit kam eine Verstärkung in Wörtern finnougrischen Ursprung mit den Stammlauten $u$ und $\ddot{u}$ infolge der Betonung zustande, die mit einer Öffnung einherging. In unbetonten Silben dagegen erfolgte in der urungarischen Zeit eine Schwächung, die mit einer Reduktion am Wortende einherging (Schließsung) und von einer Labialisierung begleitet war. Aufgrund dieses Modells kann man von einer Art Überkreuzwirkung sprechen, da in den betonten und in den unbetonten Silben jeweils entgegengesetzte Entwicklungen vonstatten gingen, deren Ergebnisse dann auf die Silben in der jeweils anderen Position gewirkt haben.
\end{abstract}

Die ungarische Lautgeschichte hat die Verschiebungen der kurzen Vokale, die sich in der urungarischen und der altungarischen Zeit vollzogen haben, mit ziemlicher Sicherheit erschlossen. Aufgrund ihrer Ergebnisse (Losonczi 19151917; Laziczius 1941-1943; Kubínyi 1958; Abaffy 1974, zusammenfassend Bárczi 1967) waren die wichtigsten Veränderungen: die Schließung der Stammendlaute, die Reduktion am Wortende, die damit zusammenhängende Labialisierung, der Wegfall der Stammendvokale und die so genannte Ersatzdehnung. Diese Prozesse sowie eventuelle weitere (Abaffy 1974, 431) sind keine individuellen Entwicklungen, sondern hängen durchaus zusammen. Dennoch ergibt sich selbst aus der wohl umfassendsten Synthese (Bárczi 1967) kein Prozess, der das gesamte System verändert und eine klar erkennbare Tendenz verkörpert. Gab es einen Grund für die Verschiebungen, den man erschließen kann? Und wenn 
ja, könnte dies die von Kubínyi vertretene Beschleunigung des Sprechtempos, die Auflösung der Intonationsrelationen gewesen sein? Lässt sich eine Tendenz feststellen, und kann man diese nach László Deme $(1968,18)$ als „Lockerung“ oder nach Loránd Benkő $(1988,149)$ als ,akustische Schwächung“ bezeichnen, kann man auf dieses Phänomen die von Erzsébet Abaffy $(1991,29)$ bezüglich der Konsonanten formulierte „Schwächung“" anwenden? Lässt sich die Öffnung, die sich in der altungarischen Zeit vollzogen hat und das gesamte System der kurzen Vokale betraf, in diesen Prozess einordnen, und wenn ja: wie? Es lohnt sich, diese Fragen immer wieder zu stellen, da eine wirklich befriedigende Gesamtdarstellung der systematischen Veränderung ungeachtet der zahlreichen Einzelbeobachtungen immer noch fehlt.

Eben deshalb wirkte das erstmals 1984 in A nyelv és a nyelvek [Die Sprache und die Sprachen] (Kenesei 1989, 172-76) publizierte Wandlungsmodell als Neuheit. Demnach hat die zu Beginn der altungarischen Zeit erfolgte Entwicklung des $i$ in zwei Richtungen $(i>u, \underline{i}>i)$ eine Serie von Verschiebungen im gesamten System ausgelöst. Aus $i$ entstandenes $u$ und $i$ müssen Homonymien mit den ursprünglicheren $u$-s und $i$-s hervorgebracht haben, zu deren Beseitigung dann eine Verschiebung dieser ursprünglicheren $u$-s und $i$-s erfolgte: $i$ wurde zu $\ddot{u}$ bzw. $\ddot{e}$, und $u$ wurde zu $o$. Dies löste selbstverständlich weitere Veränderungen der ursprünglicheren $o$-s, $\ddot{e}$-s und $\ddot{u}$-s aus. Und so weiter. Am Ende dieser Verschiebung ,wird das System insgesamt modifiziert, wobei es jedoch seinen Systemcharakter bewahrt" (176). Ein Laut (i) geht verloren, und ein neuer Laut entsteht (bzw. zwei neue Laute entstehen): das $\ddot{o}$ (und das $a$, bei dessen Herausbildung auch die Labialisierung $\dot{a}>a$ eine Rolle gespielt hat). Keneseis Verdienst besteht darin, dass er den systematischen Charakter der Entwicklung aufgezeigt hat, deren Hauptmotiv seiner Meinung nach das Bestreben darstellte, Homonymien zu vermeiden. István Kenesei hat das zunächst in einer populäreren Variante veröffentlichte Modell auch in einer wissenschaftlichen Mitteilung dargelegt (1995), die auch das Beweismaterial enthält. Dort betont er, dass das Hauptmotiv für die Lautverschiebungen die Bewahrung eines minimalen phonematischen Kontrasts war. Zur Untermauerung seiner Behauptungen führt er folgende Beispiele an: szim 'szem - Auge' und szim 'szív - saugen', visz 'visz - tragen' und visz 'vissza - zurück', bics 'becs(ül) - Wert (wertschätzen)' und bics(ak) 'Messer'. Außerdem skizziert er eine zeitliche Abfolge der Lautveränderungen mit 10 Stufen, die er zeitlich abgrenzt und 3 Perioden zuordnet. Der ersten Periode ordnet er die Entwicklung $i>i$ und $i>u$ zu (die im 12. Jahrhundert abschließst), der zweiten die Verschiebung $i>\ddot{e}, i>\ddot{u}$ und $u>o$ (die im 13. Jahrhundert abschlieft) und die Verschiebung $\dot{a}>a$ (die im 14. Jahrhundert endet), und schließlich der 
dritten die Veränderung $\ddot{e}>\ddot{o}, \ddot{u}>\ddot{o}$ und $o>a$ (wobei er zu der Entwicklung $\ddot{e}>e$ keine Stellung bezieht).

Aufgrund der systematischen Betrachtungsweise der Entwicklung der Vokale, der Erklärung der Entwicklung mit dem Bestreben nach der Vermeidung von Homonymien, weiterhin aufgrund der Tatsache, dass er die Bedeutung der trotz der Probleme hinsichtlich der Lesarten zweifelsfrei erkennbaren Öffnung in der altungarischen Zeit für die systematische Entwicklung erkannt hat, ist Keneseis Modell auf jeden Fall als eine wertvolle und nicht zu umgehende Erklärungsmöglichkeit zu bewerten. Doch auch diese äußerst gefällige Theorie wirft diverse Fragen auf. Die erste ist die nach der Position des $i$ im ungarischen Lautsystem. Dieser Laut war nämlich ein sehr seltenes Phonem (Benkô 1988, 156). Somit ist es schwer vorstellbar, dass ein so peripheres Phonem eine Wandlung dieser Größenordnung ausgelöst haben könnte. Ein weiteres Problem besteht darin, dass die Wandlung eines Lautes, der im Ergebnis mit einem bereits vorhandenen zusammenfällt, letzteren nicht unbedingt verdrängt und ebenfalls zur Wandlung zwingt. Hier ein Gegenbeispiel: Neben dem aus der finnougrischen Basissprache ererbten $w$ entwickelte sich in der ersten Hälfte der urungarischen Zeit aus intervokalischem $m$ ebenfalls $w$. Hier kann keine Rede davon sein, dass sich das bereits früher vorhandene $w$ weiterentwickelte und das aus dem $m$ entstandene bestehen blieb; im Gegenteil, sie haben sich auf identische Weise weiterentwickelt (vgl. Bereczki 1998, 71, 75). Und schließlich enthält die Theorie keine Ausführungen darüber, ob die betonte oder unbetonte Position die Art und den zeitlichen Ablauf der Veränderung beeinflusst hat.

Über die allgemeinen Vorbehalte hinausgehend kann man auch den Details nicht in allem zustimmen. Die erste Angabe für szív $(\sim$ szim) stammt von 1456, ganz zu schweigen von szimatol 'schnüffeln' (1777). Zudem findet sich laut TESz. in den finnougrischen Formen von sziv eher ein $p$, und da die Entwicklung $-p->-w$ - wahrscheinlich in der frühen urungarischen Zeit einsetzte, wurde dieses Verb am Ende der Periode vermutlich bereits mit $w$ gesprochen, weshalb die Möglichkeit einer Homonymie mit dem Nomen szim 'Auge' schwer vorstellbar ist. Auch im Fall von vissza ist die Belegung problematisch, da die erste Angabe von 1337 stammt. Am gewagtesten ist die Gegenüberstellung von becsül und bicsak. Beide sind nicht vor dem 15. Jahrhundert zu belegen, und der von Kenesei angenommene Stamm bics-für becsül lässt sich nirgendwo belegen. Die Herkunft von bicsak ist umstritten; wenn man von der italienischen Herkunft ausgeht, ist das $i$ des Wortes palatal, im Falle der türkischen Herkunft liegt das Problem in dem späten Zeitpunkt des ersten Vorkommens. Diese drei Beispielpaare können also nur mit großem Wohlwol- 
len als Homonyme einer für die Zeit vor dem 12. Jahrhundert angenommenen Synchronie angesehen werden.

Überhaupt ist es problematisch, das Ende des Wandels $\underset{i}{i} \rightarrow i$ im 12. Jahrhundert anzusiedeln, da die in der "Grabrede“ (HB) vorkommende Form achsin 'Frau' sowie die in der „Grabrede und Fürbitte“ (HBK) vorkommende Form homus 'falsch', außerdem das von 1237 belegte Ohzynfolua das Vorkommen des $i$ im 13. Jahrhundert belegen (vgl. Benkó 1980, 116). Ähnlich verhält es sich mit der rumänischen Form Ciuc (sprich: Tschuk) des Ortsnamens Csik, die laut Bárczi $(1967,144)$ frühestens aus dem 12. Jahrhundert stammen kann, während Benkô $(1990,122)$ eine noch spätere Übernahme als gesichert ansieht. Es finden sich noch weitere Beispiele bei Kenesei, die schwer zu akzeptieren sind, so z. B. bükk 'Buche' für den Wandel $i>\ddot{u}$, dessen erste Form, die mit Sicherheit labial war, von 1419 stammt (OklSz., TESz.), oder für die Verschiebung $\ddot{e}>\ddot{o}$ das Wort fö 'Haupt', das nur diphthongisch gewesen sein kann.

Kenesei datiert die Verschiebung $o>a$ auf das 13-14. Jahrhundert, Bárczi (1955, 211-13) hingegen beweist mit Hilfe der bei Konstantin vorkommenden Formen des Namens Taksony die Existenz des labialen $a$ im 10. Jahrhundert. Mit dem 13-14. Jahrhundert datiert Kenesei diese Entwicklung also mit Sicherheit zu spät (um so mehr, als sie auch in der von ihm als Quelle angegebene Bárczi-Zusammenfassung [1967, 179] auf das 10-14. Jahrhundert angesetzt wird, und nicht, wie in Keneseis Tabelle 3, auf das 13-14. Jahrhundert). Kenesei nimmt nicht nur für die Verschiebung $o>a$ das 13. Jahrhundert als frühestmöglichen Zeitpunkt an, sondern auch für das Auftreten des $\ddot{o}$. Im Gegensatz dazu ist gesichert, dass die Verschiebung $o>a$ bereits im 10. Jahrhundert und das $\ddot{o}$ mit Sicherheit schon im 12. Jahrhundert existierte (vgl. Benkô 1980, 112-6). Im Spiegel der Kritik geraten die geordneten Zeitbestimmungen von Keneseis Tabelle 3 also ordentlich durcheinander. Um nur auf die beiden Endpunkte einzugehen: Das die Veränderung auslösende $\underset{i}{i}$ war im 12. Jahrhundert noch vorhanden, und die zum Abschluss des angenommenen Prozesses entstehenden Laute $a$ und $\ddot{o}$ existierten zu dieser Zeit bereits. Somit lässt sich beim besten Willen keine geradlinige Entwicklung skizzieren, in der das $\underset{i}{i}$ der Ausgangspunkt und $a$ und $\ddot{o}$ die Endpunkte wären. Gegen eine derartige geradlinige Entwicklung spricht auch die allgemein bekannte Tatsache, dass die Sprache der altungarischen Zeit lediglich als eine mehr oder weniger lockere Einheit von Dialekten zu betrachten ist, in denen einzelne Entwicklungen je nach Dialekt unterschiedlich zur Geltung kamen.

Im Folgenden versuche ich, eine andere Möglichkeit aufzuzeigen, die im Wesentlichen auf den Traditionen der Literatur zur ungarischen Lautgeschichte basiert und nur die Akzente etwas anders setzt: Sie löst sich von der Konzen- 
tration der Entwicklung der Vokale auf die altungarische Zeit und verschiebt die Ursprünge der Entwicklung zur urungarischen Zeit hin. Die Grundlage für diese Betrachtungsweise liefern die Betonungsverhältnisse des Ungarischen (vgl. Losonczi 1915-1917, 406), und ihre Grundthese besagt, dass $a$ und $\ddot{o}$ zu Beginn der altungarischen Zeit bereits existierten. Dies versuche ich nun plausibel zu machen.

Die Meinungen darüber, ob das $a$ in der urungarischen Zeit (eventuell in der finnougrischen Grundsprache) bereits vorhanden war, gehen auseinander. Das von Steinitz für die finnougrische Grundsprache erschlossene Vokalsystem beinhaltet den labialen Velaren tiefer Zungenlage (Bereczki 1998, 38). Auch Gulya (1967) behauptet, das labiale $a$ sei in der ugrischen Grundsprache vorhanden gewesen. Wenn man sich von den mit $h$ beginnenden Einträgen des MSzFE. die Wörter hab 'Schaum', had 'Heer', haj 'Haar', hal 'sterben', hamu 'Asche', harag 'Zorn', három 'drei', hat 'sechs' und ház 'Haus' ansieht, so zeigt sich mit Ausnahme von haj bei allen o oder $u$ als Stammvokal in der grundsprachlichen Form. Die regelmäßige Entwicklung $u>o>a$ ist eine Öffnung, und diese ist mit der betonten Position des Stammvokals zu erklären. Die frühesten ungarischen Angaben für diese Wörter weisen jedoch (mit Ausnahme von hab, három und ház) das Graphem o auf. Dieses o kann man als $a$ lesen (Benkó 1980, 91-1, 110), keinesfalls aber als $\dot{a}$. Die ersten Angaben für három und ház jedoch enthalten den Buchstaben $a$ (hab ist erst sehr spät belegt, deshalb kann es hier nicht berücksichtigt werden), den man als $a$ oder $\dot{a}$ lesen kann. Die Öffnung in ungarischen Wörtern finnougrischer Herkunft, deren Stammvokal auf $u$ (o) zurückzuführen ist, hatte also zu Beginn der altungarischen Zeit $a$ oder $\dot{a}$ zum Ergebnis. (Selbstverständlich kann sich das $a$ in bestimmten Positionen, schon allein aufgrund der großen Zahl der Laute $\dot{a}$ und $a ́$ in dieser Periode, zu $\dot{a}$ weiter entwickelt haben-vgl. z. B. im Fall von hal Benkó 1980, 109). Für die Labialisierung des illabialen Velars in Wörtern, die ursprünglich ein $\dot{a}$ enthielten, kann man sich hingegen Benkôs Feststellung anschließen, dass nämlich diese Entwicklung vielleicht schon am Ende der urungarischen Zeit, mit Sicherheit aber ganz zu Beginn der altungarischen Zeit vonstatten ging (Benkő 1980, 107). Meine Schlussfolgerung lautet: Das $a$ existierte zu Beginn der altungarischen Zeit als Weiterentwicklung sowohl von $o$ als auch von $\dot{a}$. Auch András Róna-Tas datiert die Entstehung des $a$ auf die urungarische Zeit, wobei er es für bemerkenswert hält, dass sich in der mittleren bzw. unteren Wolgaregion die frühe Labialisierung des illabialen $\dot{a}$ im Türkischen nachweisen lässt, was sich aufgrund der arealen Berührungen auch auf die Entwicklung des ungarischen Vokalsystems ausgewirkt haben kann (Róna-Tas 1997, 55-6). 
Problematischer scheint die Belegung des Vorhandenseins des ö zu Beginn der altungarischen Zeit, da die Tradition der ungarischen Sprachgeschichte (und vor allem Bárczis Zusammenfassung - 1967, 178-9) quasi zum Dogma erstarrt ist. Sie besagt, dass das $\ddot{o}$ zu Beginn dieser Periode fehlte, frühestens im 13. Jahrhundert auftrat, seine Entwicklung jedoch erst im 16. Jahrhundert abgeschlossen war. Im Gegensatz dazu ist das $\ddot{u}$ - und wenn auch nur als Variante - bereits für die finnougrische Grundsprache mit Wahrscheinlichkeit anzunehmen (Bereczki 1998, 40, 78). Wo es aber $\ddot{u}$ gibt, da provoziert es geradezu die Entstehung von $\ddot{o}$ (Benkő 1988, 156). Benkő liest die Buchstaben $u$, $w, v$, die in den schriflichen Angaben des 12. Jahrhunderts vorkommen, oft als einen $\ddot{o}$-Laut $(1980,112)$. Beispiele dafür sind köz 'Gasse' und könny 'Träne'. Diese sind nämlich auf den Stammvokal $i \sim \ddot{u}$ zurückzuführen, und aufgrund der ungarischen Intonationsverhältnisse ist die Öffnung des Vokals der betonten Silbe-ebenso wie beim $a$ - auch hier mit Wahrscheinlichkeit anzunehmen.

Ich bin jedoch - anders als Benkő - der Meinung, dass bei einigen seiner Beispiele für den Buchstaben $e$ ebenfalls ein $\ddot{o}$ anzunehmen ist. In der Literatur zur Geschichte der ungarischen Rechtschreibung wird weitgehend die Auffassung vertreten, dass $e$ nicht als $\ddot{o}$ gelesen werden kann. Um diese These als gültig beibehalten zu können, konnte Bárczi nicht anders, als eine Delabialisierung in der urungarischen Zeit anzunehmen, um so z. B. die Form ketnie des ursprünglich labialen köt 'binden' (HB) erklären zu können $(1967,109)$ — wobei er sogar riskierte, die so entstandenen illabialen Formen zeitlich nach den labialen anzusiedeln (vgl. OklSz. 1138. Kuteles). Mit anderen Worten, er nahm eine Entwicklung $a>b>a$ an. Wie viel leichter wäre es aber zu akzeptieren, dass es sich an der besagten Stelle nur um eine Besonderheit der Schreibweise handelt, bei der gerade der Buchstabe $e$ das Vorhandensein des offeneren Lautes $(\ddot{o})$ im Unterschied zu dem geschlosseneren $(\ddot{u}-\mathrm{vgl}$. obiges Beispiel aus OklSz.) anzeigt! Ein bisschen weiter ausholend kann man aus einem handschriftlichen Testament vom Anfang des 16. Jahrhunderts (1517) folgendes Beispiel anführen: „... mynd menes kezeth, mynd Barom kezewt, mynd yhok kewzewt..." (Hegedús-Papp 1992, 65). Es steht außer Zweifel, dass der Urheber aus dem Komitat Tolna, der sein Testament eigenhändig abgefasst hat, die Postposition mit $\ddot{o}$ gesprochen hat, und dennoch schwankt die Rechtschreibung, d. h. für den Laut $\ddot{o}$ wird stellenweise der Buchstabe $e$ verwendet. In einem früheren Artikel (Hegedús 1989, 98-9) habe ich gezeigt, dass die Schreibung der Ortsnamen des Komitats Vas im 15. Jahrhundert bei denen, die heute mit $\ddot{o}$ gesprochen werden und wohl auch damals so gesprochen wurden, die Zeichen $e, u, o$ und $e w$ aufweist. 
Wenn man also davon ausgeht, dass die Laute $a$ und $\ddot{o}$ zu Beginn der altungarischen Zeit existierten, kann man ihre Entstehung nur auf das Ende der urungarischen Zeit datieren. Im Zuge dieses Wandels können $a$ und $\ddot{o}$ in betonten Silben entstanden sein, indem in Wörtern finnougrischen Ursprungs mit den Stammlauten $u$ und $\ddot{u}$ in der urungarischen Zeit infolge der Betonung eine Verstärkung zustande kam, die mit einer Öffnung einherging (vgl. die oben als Beispiele für Velare angeführten Wörter mit dem Anfangsbuchstaben $h$; als Beispiele für Palatale die ungarischen Wörter finnougrischen Ursprungs öl 'Schoßs', köcsög 'Krug', köd 'Nebel', könny 'Träne', könyök 'Ellbogen', köz 'Gasse', köt 'binden' und lök 'stoßen'). In unbetonten Silben dagegen erfolgte in der urungarischen Zeit eine Schwächung, die mit einer Reduktion am Wortende einherging (Schließung) und von einer Labialisierung begleitet war. Eine typische Entwicklungsrichtung der von Benkő $(1988,149)$ beschriebenen Unbetontheit ist nämlich die Labialisierung. Also geht die Entwicklung in der betonten Silbe von den geschlossenen Lauten zu den offenen und in den unbetonten umgekehrt. Im nächsten Schritt (in der nächsten Periode) ging die Labialisierung in der unbetonten Silbe, die sich in den suffigierten Formen durchgesetzt hatte, auch auf die Stammvokale über (Labialisierung nach Losonczi - vgl. Bárczi 1967, 155), während die in der betonten Silbe entstandene Öffnung auch in den unbetonten Silben zur Geltung kam. Tatsächlich kann man somit von einer Art Überkreuzwirkung sprechen, da in den betonten und in den unbetonten Silben jeweils entgegengesetzte Entwicklungen vonstatten gingen, deren Ergebnisse dann auf die Silben in der jeweils anderen Position gewirkt haben.

Die Veränderungen der Vokale in der altungarischen Zeit lassen sich als Folgen und Ergebnisse dieser Überkreuzwirkung beschreiben. Nach dem Muster der bereits vorhandenen Laute wurde im ersten Abschnitt der Periode $i$ zu $\ddot{u}$, $\ddot{e}$ zu $\ddot{o}$ und $\dot{a}$ zu a labialisiert. In diese Entwicklung lässt sich auch der Wandel $i>u$ in der unbetonten Silbe (asszony 'Frau', hamis 'falsch', Somogy 〈ein Ortsname) ) eingliedern, der mit großer Wahrscheinlichkeit ebenfalls schon zu Beginn der altungarischen Zeit wirksam war. Die andere Richtung dieser Entwicklung war, wie das auch Kenesei aufzeigt (a. a. O., 285), der Wandel $i>i$ in der betonten Silbe. Das so entstandene $i$ schob das dort vorhandene $i$ weiter (so Kenesei) oder, wenn letzteres bereits vorher labialisiert war, trat an seine Stelle. Es gibt nämlich Möglichkeiten für die Labialisierung des $i$ im Stamm in der urungarischen Zeit (Bárczi 1967, 108-9) und Beispiele dafür vom Anfang der altungarischen Zeit (Gründungsurkunde des Klosters Tihany [TA.]: cues 'steinig', fuegnes 'sandig'; bei diesen Beispielen muss man auch mit der labialisierenden Wirkung des $w$ rechnen). (Auf die Möglichkeit einer 
solchen Entwicklung weist auch Kenesei in einer Fußnote hin, in der er sich auf eine mündliche Mitteilung von Erzsébet Abaffy beruft und die Erklärung ohne Quellenangabe Gombocz zuschreibt. In Gombocz' „Lautgeschichte“ ist in der Tat die Rede davon: Gombocz nimmt für den Wandel $i>\ddot{u}$ die urungarische Zeit an [zumindest in denjenigen Dialekten, in denen diese Entwicklung erfolgte] - Gombocz 1940, 74). Ich bin der Ansicht, dass man keine dieser Annahmen vollkommen ausschließen kann. Da der Beginn der Veränderung $i>$ $\ddot{u}$ bis zum Ende der urungarischen Zeit zurück reicht (Bárczi 1967, 156), kann es Wörter gegeben haben, in denen sie schon abgelaufen war, und die bereits begonnene Entwicklung durch das aus $i$ entstandene $i$ unterstützt wurde (wobei aber auch eine direkte Entwicklung $i>\ddot{u}$ nicht kategorisch auszuschließen ist — vgl. Gósy 1998, 277).

In der zweiten Phase kam es bei den Palatalen auch in den unbetonten Silben zur Öffnung, die dann auf die Velare überging, so dass sich in den unbetonten Silben die Veränderungen $u>o$ und $o>a$ vollzogen. [Dass diese Entwicklung vielleicht zuerst bei den Palatalen einsetzte, kann man nach Bárczi $(1978,148-51)$ behaupten, auch wenn die bereits erwähnten Probleme bezüglich der Lesart der Angaben mit Velaren (Benkő 1980, 91-2, 110) diese Behauptung etwas unsicher machen.]

Es mag überraschen, dass ich die „Phasen“ nicht zeitlich definiere. Das liegt daran, dass ich ein Modell vorstellen wollte und zugleich weiß, dass die Veränderung/Entwicklung bei weitem nicht geradlinig war, sondern ein Prozess mit zahlreichen Faktoren. (Beispielsweise lässt sich die Labialisierung auch auf weitere Gründe zurückführen: Einfluss eines labialen Konsonanten, Einfluss des $l$, assimilierende Wirkung eines labialen Vokals — vgl. Egriné Abaffy 1965, 16773.) Außerdem gibt es entwicklungsgeschichtliche und entwicklungspsychologische Gründe: die neu entstandenen Formen wurden wohl neben den bereits vorhandenen gebraucht; es kann gesonderte Entwicklungen in den Dialekten gegeben haben; die Analogie kann bremsend gewirkt haben; die Entwicklung kann bei Vokalen in labialer bzw. illabialer Umgebung jeweils unterschiedlich gewesen sein; und schließlich kann die Akzeptanz von Neuerungen - darauf deuten die Untersuchungen zu den Entwicklungen in der modernen Dialektforschung hin — auch durch die Lebensweise (türkischer Einfluss) und die Bildung motiviert gewesen sein. 


\section{Literatur}

Abaffy, Erzsébet E. 1974. Szóvégrendszerünk az ôs- és az ómagyar korban. A szóvégi redukálódás [Das System der Wortenden in der ur- und der altungarischen Zeit. Die Reduktion am Wortende]. In: Magyar Nyelv $70: 430-40$.

Abaffy, Erzsébet E. 1991. Az ôsmagyar fonémarendszer történetéhez [Zur Geschichte des urungarischen Phonemensystems]. In: Mihály Hajdú - Jenő Kiss (Hg.) Emlékkönyv Benkő Loránd hetvenedik születésnapjára [Festschrift zum siebzigsten Geburtstag von Loránd Benkő.], 27-30. ELTE, Budapest.

Bárczi, Géza 1955. Az o > a nyíltabbá válás legrégibb példája [Das älteste Beispiel für die Öffnung $o>a$ ]. In: Magyar Nyelv $51: 211-3$.

Bárczi, Géza 1967. Hangtörténet [Lautgeschichte]. In: Géza Bárczi-Loránd Benkő- Jolán Berrár (Hg.) A magyar nyelv története [Die Geschichte der ungarischen Sprache], 95180. Tankönyvkiadó, Budapest.

Benkő, Loránd 1980. Az Árpád-kor magyar nyelvû szövegemlékei [Ungarischsprachige Textdenkmäler der Árpádenzeit]. Akadémiai Kiadó, Budapest.

Benkő, Loránd 1988. A történeti nyelvtudomány alapjai [Die Grundlagen der historischen Sprachwissenschaft]. Tankönyvkiadó, Budapest.

Benkő, Loránd 1990. Adalékok a székelyek korai történetéhez [Angaben zur Frühgeschichte der Szekler]. In: Új Erdélyi Múzeum 1:109-22.

Bereczki, Gábor 1998. A magyar nyelv finnugor alapjai [Die finnougrischen Grundlagen der ungarischen Sprache]. Universitas, Budapest.

Deme, László 1968. A magyar nyelv történetéhez A magyar nyelv története kapcsán [Zur Geschichte der ungarischen Sprache-anlässlich der Geschichte der ungarischen Sprache]. In: Magyar Nyelv 64:14-23.

Egriné Abaffy, Erzsébet 1965. Sopron megye nyelve a XVI. században [Die Sprache des Komitats Sopron im 16. Jahrhundert]. Akadémiai Kiadó, Budapest.

Gombocz, Zoltán 1940. Gombocz Zoltán összegyûjtött mûvei [Gesammelte Werke von Zoltán Gombocz], Band 2, Heft 1, hrsg. von Gyula Laziczius und Dezső Pais. Budapest.

Gósy, Mária 1998. Hangtörténeti változások feltételezett okairól [Über die angenommenen Gründe lautgeschichtlicher Veränderungen]. In: Magyar Nyelv 94:276-83.

Gulya, János 1967. Hodu vagy hadu? [Hodu oder hadu?]. In: Magyar Nyelv 94:276-83.

Hegedûs, Attila 1989. Helynevek a helyesírás-történet és a nyelvjárástörténet szolgálatában [Ortsnamen im Dienst der Orthographiegeschichte und der Dialektgeschichte]. In: Lajos Balogh - Ferenc Ördög (Hg.) Névtudomány és múvelődéstörténet [Namenkunde und Bildungsgeschichte], 96-9. Zalaegerszeg Város Tanácsa, Zalaegerszeg.

Hegedûs, Attila-Lajos Papp 1992. Középkori leveleink (1541-ig) [Ungarische Briefe aus dem Mittelalter (bis 1541)]. Tankönyvkiadó, Budapest.

Kenesei, István (Hg.) 1989. A nyelv és a nyelvek [Die Sprache und die Sprachen]. 2., erweiterte Ausg., Gondolat, Budapest. 
Kenesei, István 1995. Történeti nyelvtan: elmélet és gyakorlat [Historische Grammatik: Theorie und Praxis]. In: Magyar Nyelv $91: 281-91$.

Kubínyi, László 1958. Magyar nyelvtörténeti változások vélhető összefüggéséról [Ü̉ber den angeblichen Zusammenhang ungarischer sprachhistorischer Wandlungen]. In: Magyar Nyelv $54: 213-32$.

Laziczius, Gyula 1941-1943. Egy nagy pör felújítása [Die Wiederaufnahme einer großen Streitfrage]. In: Nyelvtudományi Közlemények $51: 241-79$.

Losonczi, Zoltán 1915-1917. Az ö-zés története [Die Geschichte der ö-Dialekte]. In: Nyelvtudományi Közlemények $44: 373-406$.

Róna-Tas, András 1997. Török nyelvi hatások az ősmagyar nyelvre [Türkische Spracheinflüsse auf das Urungarische]. In: László Kovács - László Veszprémi (Hg.) Honfoglalás és nyelvészet [Landnahme und Sprachwissenschaft], 49-60. Balassi Kiadó, Budapest.

\author{
Adresse des Verfassers: Attila Hegedûs \\ 1062 Budapest \\ Aradi u. 16. \\ Ungarn \\ hegedus@btk.ppke.hu
}

